\title{
A COLLECTIVE AWARENESS PLATFORM FOR MISSING CHILDREN INVESTIGATION AND RESCUE
}

\author{
Ariadni Michalitsi-Psarrou, Christos Ntanos and John Psarras \\ National Technical University of Athens, Athens, Greece
}

\begin{abstract}
The plight of missing children is particularly strenuous and sensitive for society at local, national and European levels, cutting across class, race, and age. A quarter million cases of missing children is reported in the EU annually, which are either parental abductions, stranger abductions, runaways, missing unaccompanied migrant minors, and generally lost, injured, or otherwise missing children. The problem of missing children is a complex, multi-faceted phenomenon, comprising legal, psychological and sociological aspects, which are complicated further due to the strong emotions from the close environment of the missing child. This paper presents the challenges missing children investigation and rescue currently faces, and proposes a solution that uses ICT, advanced analytics and collective intelligence, to achieve more rapid and effective resolutions. The proposed methodology leverages the untapped potential of open, social, and linked data to augment the background information of missing children, through multi-layer - personal, psychological, social and activity - profiling and predictive analytics, respecting and protecting privacy, and personal data. Using location-based mobile notifications that spread using geo-fencing, citizens close to the place a missing child was last seen or is more probable to be found become "social sensors" for the investigation, contributing and validating potential pieces of evidence. Through the EU-funded project ChildRescue, the proposed solution is currently at the last phase of its development and aims to be adopted by different voluntary organisations, according to their needs and the readiness of their systems and processes. The project's results are now piloted in missing children cases by organisations responsible for the Amber Alert, and the 116 000 pan-European hotline, as well as unaccompanied minors' cases supported by the Hellenic Red Cross. The resulting collaboration platform and mobile applications will be publicly launched in 2020 .
\end{abstract}

\section{KEYWORDS}

Missing Children, Collective Awareness, Profiling, Analytics, Crowdsourcing, Unaccompanied Migrant Minors

\section{INTRODUCTION}

While the phrase "going missing" regarding a child is generally understood as the occurrence of a situation characterised by a lack of information on the whereabouts of that child, each country tends to adopt its own, nonbinding definition of what constitutes a missing child. These definitions have significant variations to the risk posed, the cause of the disappearance and the existence (or not) of indications that a crime is being or has been committed, leaving it up to the authorities to decide instantly how an individual case should be handled and whether it should be registered as referring to a missing child or not (Best 1987). Such a process often introduces delays in the crucial early stages of a disappearance, especially if an official report should have been filed to declare a disappearance so as to trigger an official alert (Cancedda et al. 2013; Griffin et al. 2007; Sicafuse and Miller 2010).

A special missing children case, that in the recent years has become increasingly and alarmingly pertinent, is that of unaccompanied refugee minors. The lack of family, which is usually, at least geographically, out of reach, as well as the lack of other reliable social networks, renders their position highly insecure and vulnerable. Paradoxically, despite the enormous risks to which unaccompanied refugee children are exposed and the fact that all actions and laws which apply to missing children, in general, equally apply to unaccompanied migrant children, their disappearance is usually underreported, strengthened by the fact that some European countries have employed mechanisms that give these cases an automatic lower priority than other missing children, by imposing, for instance, a mandatory waiting period before filling a missing person report (Cancedda et al. 2013). Research reveals that up to $50 \%$ of the unaccompanied children accommodated go missing from 
reception centres in the European Union, and in many cases, information about the child's whereabouts remains scarce (available at: http://missingchildreneurope.eu/).

Currently, missing children alerts, patterned after the Amber Alert System, have been implemented in many European countries (named either Amber or Child Alert), while more and more EU countries develop similar alert systems. These systems aim to quickly disseminate critical information about particularly worrying child disappearances to the public through a variety of channels, thus increasing the probability of a positive outcome. The idea behind the Amber/Child Alert System is that, by broadcasting and distributing information about a missing child to the community, the public's involvement can trigger critical feedback that would have otherwise been ignored, and which can prove critical in finding the missing child (Griffin 2010). It is launched only in exceptional cases, the reason being that too frequent alerts would lead to lack of interest by the public. It has been claimed that identification times have been reduced for a high number of missing children cases thanks to information provided by citizens aware of the issued amber alerts, leading to an estimated effect of more than $62 \%$ of cases resolved based on citizens' tips or discovery of the missing person (Griffin 2010; Kamel Boulos et al. 2011). At the same time, the effectiveness of these systems has been criticised by several researchers, especially on what concerns the claim for high annual numbers of 'saved lives', due to intrinsic difficulties with the evaluation of the effectiveness of these alerts (Griffin et al. 2016; Griffin and Miller 2008; Sicafuse and Miller 2010). It is, indeed, a reasonable consideration that since it would be immoral to form a control group to validate the impact of the Amber Alert, by selecting cases where alerts should have but aren't issued, a scientific measurement for the claim for "saved lives" and the reduction of time needed to locate a missing child becomes very difficult.

\section{CHALLENGES AND OPPORTUNITIES}

Despite the remarkable efforts at state and European level to effectively address the issue of missing children, several concerns regarding the effectiveness of Amber Alert system are raised and several key challenges remain open. Clues coming from concerned citizens are instrumental to locating missing children, yet most of the information typically originates from recorded phone calls, often without adequate supporting evidence, which may derail the investigation. The ability of people to remember faces from pictures and descriptions and be able to recognise them after just a little while has been questioned by a significant body of research (Frowd, Erickson, and Lampinen 2014; Lampinen, Curry, and Erickson 2016; Sweeney and Lampinen 2012). Limits of people's cognitive functions and memory retrieval errors, such as the "tip-of-the-tongue" phenomenon, may hinder the AMBER Alert System's success, despite a person's intention to remember information from the Amber Alert and his willingness to contribute to the cause (Miller and Clinkinbeard 2006). The development of new systems that offer a real-time, two-way channel, from and to members of the community, that would allow flow of information at any time, could be proven beneficial in minimising these effects.

The lack of a mechanism that makes use and merges all available sources of information is also a challenge faced. While the information received by the relatives and the close social environment of a missing child is always the starting point for an investigation, other sources of information can prove of equal or greater value. This includes information that can be deduced from social media activities and networks of friends, as well as information coming from open data sources, such as the transportation infrastructure, road networks, or land use, which may enrich the information available and improve predictive capabilities regarding the whereabouts of the missing child (Ledoux and Van Oosterom 2013; Yuan, Zheng, and Xie 2012), resulting in an informed behavioural and activity profile of the child. According to current literature and practices (Crosland et al. 2018; Johnson, Rew, and Kouzekanani 2006; Payne 1995), the common approaches to profiling for a missing person are mostly analyses of the person's personal data and psychosocial characteristics by domain experts. The conclusions are drawn from testimonials and interviews and are of a qualitative nature. Therefore, there is no systematic way to apply different profiling techniques for recognising activity patterns of missing persons. The research challenge here is to perform semiautomated behavioural profiling for missing children.

Further to the information sources above, past cases of missing children could also be used to identify correlations and common patterns. This can be performed by applying pattern recognition techniques, already known in other application fields. Finally, since important clues may be coming from multiple witnesses' reports, these can be combined and incorporated into the investigation so as to focus on possible time-sensitive routes, which is only currently done manually, sparsely, and based on human intuition. 
Identifying the potential location of a missing person is a very complex task, requiring all sources of related information to be analysed as quickly as possible. The result of this process should be a case-specific prediction of geographic areas where the investigation and/or alerts should focus.

What is more, it is widely acknowledged that time is of the essence in the investigation of missing children, and the first hours after a disappearance are of vital importance. Although the alerts for missing children are currently quickly broadcasted on media, there is no mechanism to focus and intensify the calls for help to part of the community based on its proximity to the point the person was last seen at and other high priority areas. Efficient mobilisation of the local community is therefore usually accomplished by distributing flyers and putting up posters in these areas - a slow process - while SMS messages notifying volunteers based on their registration address are sometimes used, but don't take into account their current location and also do not contain enough details or multimedia content. Propagating localised real-time notifications to citizens (Rahate and Shaikh 2016) according to the output of predictive analytics and profiling for missing persons does not have a precedent in the international research and practice.

Lack of cross-border cooperation between institutions, law enforcement agencies and networks for missing children is also a critical issue, especially in cases of disappearances of unaccompanied migrant minors. Different national legal strategies in dealing with missing unaccompanied migrant minors further reinforce complications in cross-border cases. There is therefore a strong need for tools supporting a fast cross-border exchange of information on missing unaccompanied minors, as well as a reliable way to cross-reference and identify a child that moves from one hosting facility to another, in order to improve migration data and the misallocation of search efforts.

Dealing with these challenges in a unified and effective way requires significant research towards effectively exploiting and guiding the spontaneous network effect, by combining multiple sources of information and applying intelligent data analysis and prediction techniques. Such a novel, well-thought-out process could be considered the key to achieving faster and better conclusions to missing children's investigations.

\section{THE CHILDRESCUE SOLUTION}

To meet these fundamental challenges, this paper presents the ChildRescue solution, which develops a unique, unified approach and an applicable solution aspiring to have an outstanding impact in missing children's discovery, while also covering one of the greatest issues related to the refugees' crisis, the disappearance of unaccompanied children and the additional difficulties for identifying them. The result is a novel methodology for accelerating and amplifying the network effect and harnessing collective intelligence in the investigation of missing and unaccompanied children by creating an uninterrupted cycle of information flow and communication between all stakeholders, achieved by working on top of social theories and identifying how they can be influenced by novel ICTs towards addressing a critical societal challenge.

The ChildRescue solution aims at effectively and quickly raising collective awareness and instigating instant community action during the investigation of missing children. The phases of the missing person investigation cycle, on which the ChildRescue focus lies, include: (a) Preparation in order to lead the investigation to proper directions from its onset, (b) Coordination, with new models for collaboration among the missing children response organisations, (c) Action in order to motivate society to seamlessly contribute to the research, (d) Archiving of the case in privacy-respectful way. 


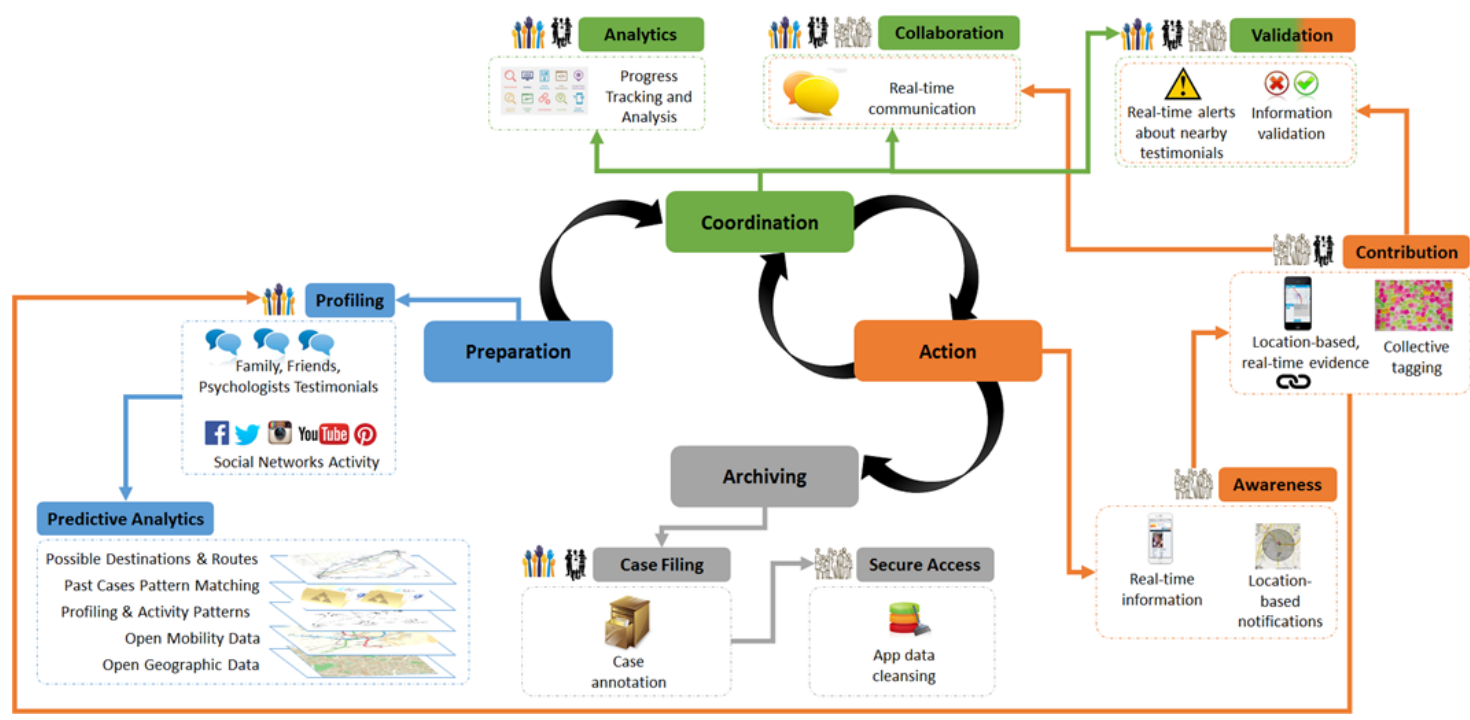

Figure 1. ChildRescue concept

Figure 1 presents the overall ChildRescue concept and solution, which is formed and cultivated around the four aforementioned phases of the missing children investigation cycle. In particular, during the investigation preparation phase, ChildRescue solution augments the information collected by the missing children response organisations by researching and employing advanced profiling methods towards the construction of a multilayer -personal, psychological, social - profile of the missing child or the unaccompanied minor. To accomplish that goal, system theories, such as Activity Theory and Collective Behaviour Theory, are analysed along with methods and scientific fields stemming from the computer science, such as Social Network Analysis and Machine Learning for modelling human behaviour, to construct reliable activity and behaviour profiles of children from both a technological and a sociological perspective. Profiling information of the missing child is then combined with open data, like geographic and transportation data, along with insights and patterns revealed from pattern matching of similar past cases, to deduce potential Points of Interest (POIs) and consequently routes and directions that the investigation should follow, in an evidence-based and scientifically-sound manner, the preliminary findings of which approach can be found in (Michalitsi-Psarrou et al. 2019).

During the coordination phase, the ChildRescue solution allows search and rescue team members to gather and contribute to the investigations under the coordination of the responsible authorities. Through a collaboration space, real-time sharing of information and communication among team members who operate in the same field is offered, in a privacy-aware manner, while an analytics interface is offered to support investigation progress tracking. A real-time interactive map is also provided, showing the location of individuals and teams on the field, allowing for better operation, monitoring, and guidance. The collaboration spaces can help organisations build a team for an investigation quickly, enhance intercommunication and enable case managers to provide guidance and support, having a complete picture of the investigation progress and its updates.

Then, in the investigation action phase, the momentum for collective intelligence in the missing child cause is built through an intuitive mobile application. Real-time and evidence-based mobile alerts and updates are broadcasted to all citizens that have the app installed on their mobile phones, but are shown using geo-fencing techniques (Garg et al. 2017), only to those in areas designated to be close to the place where the child was last seen or in a specified radius from identified POIs stemming from the analysis in the preparation phase. With the advent of time, new POIs can be extracted from the validated pieces of evidence contributed by the citizens, and thus new notifications will be diffused. Any citizen, having the application installed, may send feedback, along with potential evidence (e.g., photo). This feedback is then algorithmically assessed on the credibility of the sender, based on his past behaviour and completeness of his profile, as well as the quality of the evidence they provided. The reliability of the evidence receives an automatic provisional classification by comparing its content, its location and the time that it was captured and sent, to the current case data. Through this process, the consistency of reported information in missing children cases increases and critical information is not only 
brought to the attention of the responsible authorities, but is also spread almost in real time, through updated geo-fenced alerts.

Finally, in the archiving phase, ChildRescue supports the responsible authorities to handle the case's closure in a secure and privacy-respectful manner. Semantic annotation of the documentation and lessons learnt for each case are kept inside the organisations, in order to support similar cases in the future. Regarding the public mobile app, all data related to a case are completely erased.

From an end-user's perspective and considering the application of the above-presented ChildRescue concept in action, the ChildRescue solution is composed of two main technological elements. Firstly, a user-friendly mobile application is offered to the citizens, who will have a direct communication channel to contribute eyewitness reports. Secondly, a collaboration platform will be at the disposal of the response organisations and the search and rescue teams, offered in the form of intuitive management and coordination interfaces. From a technological perspective, on the other hand, in order to deliver the functionalities envisaged in the ChildRescue concept, the ChildRescue solution consists of several components, such as the "Collaboration Space", mentioned before, allowing all investigators to securely share location-based, multi-media information regarding the investigation in groups with specific access rights, as well as the "Notification Engine", providing a scalable and flexible mechanism for managing and delivering mobile notifications, based on applied rules (e.g., the radius around POIs, duration of notification), and the "Analytics" engine, providing real-time insights to the organisations about each case under investigation. Adopting a modular design approach, each of the components exposes the necessary functionality for its integration in the overall ChildRescue solution under an Application Programming Interface (API) that is built according to stateof-the art practices and standards.

\section{APPLICATION}

The ChildRescue solution is currently tested against two different use cases and respective scenarios. The first use case examines the emergency response to reported child disappearances and is explored by two different pilot response organisations, one in Greece, coordinated by the Smile of the Child, and the other in Belgium, by Child Focus. Both organisations are the official response organisations for missing children in their respective countries and members of the Missing Children Europe federation. The second use case engages with and supports the registration, tracing, and discovery in case of a disappearance of unaccompanied migrant minors from hosting facilities and is coordinated by the Hellenic Red Cross. The ChildRescue platform aims to be used in parallel to Red Cross's mechanisms contributing to the prevention of disappearance, improving time-management and providing a tool that will reinforce coordination, decision making and information sharing on different organisational levels (e.g., hosting facilities, regional Red Cross Departments).

By now, the first Alpha versions of the ChildRescue platform and mobile app have been released, while a simulation/tabletop exercise has run for all the three organisations. The developed scenarios were based on actual past cases to create a plausible environment, but any personal data related to the children were carefully anonymised. Members and staff of the pilot organisations participated in the exercise, highlighting several benefits, compared to the established processes, most importantly the enhancement of fast information exchange with the alerts and team coordination tools, the simplification of information transfer between hosting facilities, the promotion of public engagement and the easier identification of irrelevant incoming information. The participants' feedback is going to be used for the refinement of the public release of the ChildRescue platform, which is planned for May 2020.

Along the development and testing procedures, a set of interviews with different stakeholders and domain experts has been conducted to enrich technical expertise and research findings. The results of these interviews have exposed the needs of and challenges faced by missing children, both by the experience itself, by being away from their families, and have highlighted the great potential for preventive and intervention strategies. Combining the expertise of heterogeneous parties and creating a mutual understanding of the particularities when designing, implementing and running new processes was, though, challenging and enlightening. Applying the insights from the feedback acquired, enables a more efficient data analytics process, and the building of an enhanced, complete profile of a missing child case, that extends simple case description and attempts to explain also child's social and psychological motivations. Since the project's development and testing is still in progress, valuable feedback and insights are expected to from the field exercises that will take 
place in the following months, as well as the use of the platform in production afterwards, that we aspire to further validate its effectiveness. On what is more, a robust exploitation plan is being developed, to ensure wide exploitation and utilisation of the ChildRescue solution after its release, building, amongst others, on the Missing Children Europe's (MCE) wide network, being the umbrella organisation of all missing children response organisations in the EU, and its extensive experience in communicating activities.

\section{CONCLUSION}

This paper investigated the current challenges and opportunities in missing children investigations and rescue efforts, while presenting a possible solution to enabling faster and more effective resolution of missing children cases, including those that relate to the registration, profiling and tracing of unaccompanied migrant minors. The proposed solution builds on top of existing, state of the art social theories and technological solutions and combines them to generate added value for missing children organisations towards creating a market-ready technological offering, that will work in parallel with their existing processes.

Several key take-aways can be elicited at this point. Firstly, given the highly sensitive nature of the data needed to create activity and behaviour profiles for the missing children, data privacy and protection needs to be at the forefront of such a solution's concerns and priorities. For this reason, encryption and anonymisation techniques are employed, and GDPR compliance is ensured. Moreover, the requirement for GDPR compliance has also seriously affected the availability of data, thus restricting the research on human behaviour, mobility and activity analysis, that intrinsically relies upon public personal data. Although it is undoubtedly for the best interest of individuals, a balance between data security and scientific progress is needed, while it is still a period of adjustment. It needs to be stressed, also, that each child is unique, rendering the issue of missing children quite diverse - as highlighted by social workers - a tiny, unpredictable detail may affect a child's current mindset and turn a case towards an erratic course of events. Therefore, a completely automatic process based on analytics insights and computations cannot be a substitute of the expertise of social workers, psychologists, and other trained professionals.

Although there are several private and public organisations all over the world that have developed or adapted systems for designing and managing missing persons cases (e.g., NamUs accessible at https://www.namus.gov/Dashboard, and accessible https://www.iosb.fraunhofer.de/servlet/is/19908/), there is no inclusive solution, to the best of our knowledge, for addressing the complexity of missing children issue. The novelty of the proposed solution can be summarised in three axes. The first is the distribution of alerts based on the users' proximity to points of interest for a case, instantly grabbing the attention of several citizens at the same time, and the collection of their feedback for the development of an almost real-time and evidence-based profile of the missing child. The second axis is the facilitation of the information exchange between organisations acting on the field. The final axis is the development of data analytics and machine learning algorithms for the analysis of behavioural profiles, and their inclusion in existing investigative processes for missing children, research on which is currently limited (Blackmore et al. 2005; Lin and Goodrich 2010).

A remaining open research question concerns the validation of the success of such a process, for the same reasons that Amber Alert's success is questioned and difficult to be verified. In that respect, we consider a scientific validation feasible through a realistically simulated environment, taking into account the special characteristics of both the missing children cases and the mobility characteristics and particularities of the citizens of the wider geographical area where the child was last seen, who contribute as social sensors in the investigation,. This would provide the opportunity to run experiments comparing different alerting systems, as well as investigating process methods, in identical experimental cases, by defining baselines and controls. In that view, future work includes the development of such a simulation framework that will serve as evidence of the effectiveness of different alert systems, or lack thereof, and ChildRescue's own methodology.

\section{ACKNOWLEDGEMENT}

This work has been co-funded by the European Union's Horizon 2020 Research and Innovation programme under the ChildRescue project, grant agreement No 780938. 


\section{REFERENCES}

Best, J., 1987. "Rhetoric in Claims-Making: Constructing the Missing Children Problem." Social Problems 34(2): 101-21. https://academic.oup.com/socpro/article-lookup/doi/10.2307/800710 (January 22, 2020).

Blackmore, K. et al, 2005. "Data Mining of Missing Persons Data." In Springer. Berlin, Heidelberg, 305-14. http://link.springer.com/10.1007/11011620_19 (December 18, 2018).

Cancedda, A. et al, 2013. "Missing Children in the European Union Mapping, Data Collection and Statistics." Luxembourg:Publications Office of the European Union: 106.

Crosland, K. et al, 2018. "Why Youth Run: Assessing Run Function to Stabilize Foster Care Placement." Children and Youth Services Review 85: 35-42.

Frowd, C. et al, 2014. "Locating Missing Persons Using Age-Progression Images from Forensic Artists." In Proceedings 2014 International Conference on Emerging Security Technologies, EST 2014, Institute of Electrical and Electronics Engineers Inc., 1.

Garg, A. et al, 2017. "Smart Geo-Fencing with Location Sensitive Product Affinity." In Proceedings of the 25th ACM SIGSPATIAL International Conference on Advances in Geographic Information Systems - SIGSPATIAL'17, New York, New York, USA: ACM Press, 1-10. http://dl.acm.org/citation.cfm?doid=3139958.3140059 (January 22, 2019).

Griffin, T. and Miller, M., 2008. "Child Abduction, AMBER Alert, and Crime Control Theater." Criminal Justice Review 33(2): 159-76. http://journals.sagepub.com/doi/10.1177/0734016808316778 (February 13, 2019).

Griffin, T. et al, 2007. "A Preliminary Examination of AMBER Alert's Effects." Criminal Justice Policy Review 18(4): 378-94. http://journals.sagepub.com/doi/10.1177/0887403407302332 (January 22, 2020).

Griffin, T. et al, 2010. “An Empirical Examination of AMBER Alert 'Successes."' Journal of Criminal Justice 38(5): $1053-62$.

Griffin, T. et al, 2016. "Does AMBER Alert 'Save Lives'? An Empirical Analysis and Critical Implications.” Journal of Crime and Justice 39(4): 490-511.

Johnson, R. J. et al, 2006. "Gender Differences in Victimized Homeless Adolescents." Adolescence 41(161): 39-53. http://www.ncbi.nlm.nih.gov/pubmed/16689440 (January 28, 2020).

Boulos, M. N. K. et al, 2011. "Crowdsourcing, Citizen Sensing and Sensor Web Technologies for Public and Environmental Health Surveillance and Crisis Management: Trends, OGC Standards and Application Examples." International Journal of Health Geographics 10(1): 67. http://www.ij-healthgeographics.com/content/10/1/67.

Lampinen, J. et al, 2016. "Prospective Person Memory: The Role of Self-Efficacy, Personal Interaction, and Multiple Images in Recognition of Wanted Persons.” Journal of Police and Criminal Psychology 31(1): 59-70.

Ledoux, F. and Van Oosterom, H., 2013. "Transportation Mode-Based Segmentation and Classification of Movement Trajectories)." International Journal of Geographical Information Science 27(2): 385-407. http://dx.doi.org/10.1080/13658816.2012.692791 (February 7, 2019).

Lin, L. and Goodrich, M., 2010. "A Bayesian Approach to Modeling Lost Person Behaviors Based on Terrain Features in Wilderness Search and Rescue." Computational and Mathematical Organization Theory 16(3): 300-323. http://link.springer.com/10.1007/s10588-010-9066-2 (February 13, 2019).

Michalitsi-Psarrou, A. et al, 2019. "Complementing Amber Alert: Increasing the Social Sensors' Effectiveness through Focused Communication Channels." In Institute of Electrical and Electronics Engineers (IEEE), 1-7.

Miller, M. and Clinkinbeard, S., 2006. "Improving the AMBER Alert System: Psychology Research and Policy Recommendations." Law \& Psychology Review 30. https://heinonline.org/HOL/Page?handle=hein.journals/lpsyr30\&id=5\&div=\&collection= (February 13, 2019).

Payne, M., 1995. "Understanding 'Going Missing': Issues for Social Work and Social Services." The British Journal of Social Work 25(3): 333-48. https://academic.oup.com/bjsw/article/25/3/333/1665718/Understanding-Going-Missingissues-for-social-work (January 28, 2020).

Rahate, S. and Shaikh, M., 2016. "Geo-Fencing Infrastructure: Location Based Service.” International Research Journal of Engineering and Technology. www.irjet.net (January 28, 2020).

Sicafuse, L. and Miller, M., 2010. "Social Psychological Influences on the Popularity of Amber Alerts." Criminal Justice and Behavior 37(11): 1237-54. http://journals.sagepub.com/doi/10.1177/0093854810379618 (January 22, 2020).

Sweeney, L., and Lampinen, J., 2012. "The Effect of Presenting Multiple Images on Prospective and Retrospective Person Memory for Missing Children." Journal of Applied Research in Memory and Cognition 1(4): 235-41.

Yuan, N. J. et al, 2012. "Discovering Regions of Different Functions in a City Using Human Mobility and POIs." Proceedings of the ACM SIGKDD International Conference on Knowledge Discovery and Data Mining. pp.186-194. 